ORIGINAL ARTICLE

\title{
Historical limitations of determinant based exposure groupings in the rubber manufacturing industry
}

\author{
R Vermeulen, H Kromhout
}

Occup Environ Med 2005;62:793-799. doi: 10.1136/oem.2004.016329

See end of article for authors' affiliations

....................

Correspondence to: Dr R Vermeulen, Occupational and Environmental Epidemiology Branch, Division of Cancer Epidemiology and Genetics, National Cancer Institute, $\mathrm{NIH}, \mathrm{DHHS}, 6120$ Executive Boulevard, Rockville, MD 20892, USA; vermeulr@mail.nih. gov

Accepted 18 May 2005

\begin{abstract}
Aims: To study the validity of using a cross-sectional industry-wide exposure survey to develop exposure groupings for epidemiological purposes that extend beyond the time period in which the exposure data were collected.

Methods: Exposure determinants were used to group workers into high, medium, and low exposure groups. The contrast of this grouping and other commonly used grouping schemes based on plant and department within this exposure survey and a previously conducted survey within the same industry (and factories) were estimated and compared.

Results: Grouping of inhalable and dermal exposure based on exposure determinants resulted in the highest, but still modest, contrast $(\epsilon \sim 0.3)$. Classifying subjects based on a combination of plant and department resulted in a slightly lower contrast $(\epsilon \sim 0.2)$. If the determinant based grouping derived from the 1997 exposure survey was used to classify workers in the 1988 survey the average contrast decreased significantly for both exposures $(\epsilon \sim 0.1)$. On the contrary, the exposure classification based on plant and department increased in contrast (from $\epsilon \sim 0.2$ to $\epsilon \sim 0.3$ ) and retained its relative ranking overtime. Conclusions: Although determinant based groupings seem to result in more efficient groupings within a cross-sectional survey, they have to be used with caution as they might result in significant less contrast beyond the studied population or time period. It is concluded that a classification based on plant and department might be more desirable for retrospective studies in the rubber manufacturing industry, as they seem to have more historical relevance and are most likely more accurately recorded historically than information on exposure determinants in a particular industry.
\end{abstract}

$\mathrm{T}$ he development of exposure groups is an important component of the exposure assessment process within large (retrospective) occupational epidemiological studies. Assessing exposures to groups, rather than to individuals, is generally more efficient because individual assessments require multiple measurements on all or most of the study subjects (a situation rarely found) and because it generally results in less attenuation of the exposure-response relationship. ${ }^{1}$ As a result, especially in retrospective cohort studies, workers are often grouped based on their affiliation with certain observable work characteristics, like plant or department, with the intend to group subjects with similar exposures together. Prais and Aitchinson ${ }^{2}$ recognised that maximising the between-group variance and minimising within-group variance optimises the grouping strategy. This observation, often referred to as contrast, is a prerequisite for the detection of any exposure-response relationship in epidemiological analyses. Despite the widespread use of grouping strategies, there is so far limited experience with optimisation of these strategies within occupational epidemiological research. ${ }^{3-7}$

In 1995, Kromhout and Heederik ${ }^{3}$ reported on the efficiency of different grouping schemes for dust and dermal exposure in the rubber industry and concluded that a grouping into low, medium, and high exposure based on identified exposure determinants (that is, determinant based grouping) was the best classification for both inhalable dust and dermal cyclohexane soluble matter (CSM) exposure. The applicability of determinant based groupings in retrospective studies, however, might be hampered by the limited availability of such detailed information in the past, while for instance job histories and department affiliation are most of the time available. In addition, it is unclear how stable exposure determinants are overtime as production processes and actual task contents are constantly changing. It can therefore be questioned whether a determinant based grouping derived from a cross-sectional survey can be used to construct an exposure grouping that has any historical relevance and as such can be applied retrospectively.

We performed a follow up study, nine years after the study described by Kromhout and Heederik ${ }^{3}$ in predominantly the same factories, utilising the same measurement strategy and methodologies. ${ }^{8}$ We applied mixed-effects models to identify work characteristics that affect inhalable dust and dermal CSM exposure levels. Consequently, these exposure determinants were used to group subjects into low, medium, and high exposure groups. Contrast in average exposure resulting from this grouping and of groupings based on plant and department within the newly collected exposure data was estimated. Subsequently, the (historical) validity of the exposure grouping was determined by applying these groupings to the 1988 exposure survey. The rationale behind this exercise was to study the validity of using a contemporary cross-sectional industry-wide exposure survey to develop exposure groupings for epidemiological purposes that extend beyond the time period in which the exposure data were collected.

\section{METHODS}

Exposure information from two large industry-wide surveys conducted in the Netherlands in 1988 and 1997 was used. Characteristics of these surveys have been described in detail elsewhere. ${ }^{8-10}$ Results of the empirical exposure modelling of the 1988 data have been described in two earlier reports. ${ }^{10}{ }^{11}$ Companies involved in the two exposure surveys formed a representative cross-section of the rubber manufacturing industry in the Netherlands. The 1988 and 1997 survey comprised a total of 10 and 9 factories, respectively, of which 
Table 1 Inhalable dust and dermal CSM concentrations by department, plant, and type of plant in 1997

\begin{tabular}{|c|c|c|c|c|c|c|c|c|}
\hline & \multicolumn{4}{|c|}{ Inhalable dust $\left(\mathrm{mg} / \mathrm{m}^{3}\right)$} & \multicolumn{4}{|c|}{ Dermal CSM $\left(\mu \mathrm{g} / \mathrm{cm}^{2}\right)$} \\
\hline & $\mathbf{n}$ & AM & GM & GSD & $\mathbf{n}$ & AM & GM & GSD \\
\hline \multicolumn{9}{|l|}{ Department } \\
\hline Compound and mixing & 72 & 2.09 & 1.04 & 2.74 & 76 & 104 & 40 & 3.22 \\
\hline Pre-treating & 66 & 1.05 & 0.67 & 2.60 & 69 & 32 & 25 & 2.04 \\
\hline Moulding & 165 & 2.26 & 0.67 & 3.49 & 177 & 101 & 37 & 3.45 \\
\hline Curing & 147 & 0.68 & 0.54 & 1.77 & 153 & 121 & 40 & 3.28 \\
\hline Finishing & 64 & 2.04 & 0.72 & 2.64 & 69 & 54 & 26 & 2.74 \\
\hline Shipping & 32 & 0.66 & 0.63 & 1.37 & 33 & 109 & 35 & 3.48 \\
\hline Engineering service & 55 & 1.41 & 0.64 & 2.85 & 56 & 545 & 68 & 5.40 \\
\hline Laboratory & 12 & 0.23 & 0.21 & 1.57 & 14 & 61 & 29 & 3.23 \\
\hline \multicolumn{9}{|l|}{ Plant } \\
\hline Plant 1 & 56 & 1.01 & 0.76 & 1.91 & 54 & 198 & 38 & 3.91 \\
\hline Plant 2 & 49 & 1.23 & 0.74 & 2.34 & 52 & 212 & 50 & 3.38 \\
\hline Plant 3 & 49 & 0.53 & 0.48 & 1.58 & 53 & 119 & 44 & 3.48 \\
\hline Plant 4 & 53 & 0.92 & 0.71 & 1.83 & 54 & 153 & 43 & 3.99 \\
\hline Plant 5 & 106 & 3.93 & 0.84 & 4.98 & 111 & 93 & 39 & 3.64 \\
\hline Plant 6 & 85 & 0.83 & 0.56 & 2.24 & 99 & 89 & 45 & 2.85 \\
\hline Plant 7 & 58 & 1.90 & 0.85 & 2.76 & 62 & 238 & 28 & 3.85 \\
\hline Plant 8 & 100 & 0.71 & 0.44 & 2.11 & 105 & 122 & 28 & 3.02 \\
\hline Plant 9 & 57 & 1.11 & 0.76 & 2.25 & 57 & 39 & 26 & 2.37 \\
\hline \multicolumn{9}{|l|}{ Type of plant } \\
\hline Retreading & 57 & 1.11 & 0.76 & 2.25 & 57 & 39 & 26 & 2.37 \\
\hline Tyre & 291 & 1.92 & 0.60 & 3.24 & 315 & 102 & 37 & 3.22 \\
\hline General rubber goods & 265 & 1.14 & 0.70 & 2.15 & 275 & 186 & 39 & 3.75 \\
\hline All & 613 & 1.50 & 0.66 & 2.69 & 647 & 132 & 37 & 3.38 \\
\hline
\end{tabular}

7 participated in both surveys. Of the three companies that did not take part in the second survey, one refused to participate due to a major reorganisation, and two companies did not participate as they had merged and subsequently moved to another location. The new holding, which used the machine inventory of the old companies, did however participate in the new survey. One additional company was added to the 1997 survey to have a more representative sample of the rubber manufacturing industry in the Netherlands.

\section{Identification of exposure determinants}

Exposure data from the 1997 survey were used to identify exposure determinants of inhalable dust and dermal CSM exposure. Personal inhalable particulate exposure was measured on three consecutive days (Tuesday-Thursday) during one week by means of a PAS6 sampling head mounted near the breathing zone of the worker. ${ }^{12}$ Personal dermal exposure to cyclohexane soluble matter (CSM) was measured by means of a dermal pad sampler worn at the lower part of the wrist of the hand of preference on the same days as the personal inhalable exposure measurements were taken. ${ }^{8}$ Detailed information on tasks performed and the use of control measures (for example, gloves, local exhaust ventilation (LEV)) during the measurements was collected by standardised in-person interviews of the worker after the work shift had ended.

All exposure data and auxiliary information collected were used in linear regression models using standard stepwise regression techniques. All variables were entered in the model and were retained if the significance level was below 0.2 . Model adequacy was tested with standard diagnostic techniques such as residual plots and outlier detection. After this initial step the identified determinants (that is, tasks, local exhaust ventilation, glove use) in the stepwise regression were subsequently entered into a mixed model. Determinants were retained in the mixed model if the significance level was below 0.1. Akaike's information criterion (AIC) was used to determine the optimal combination of exposure determinants in the mixed model. Exposure determinants were treated as fixed effects in the mixed models, whereas worker and day (error) were treated as random effects (model 1 ):

$$
\mathrm{Y}_{\mathrm{ij}}=\mu+\beta_{1 . . \mathrm{k}}+\chi_{\mathrm{i}}+\epsilon_{\mathrm{ij}}
$$

$\mathrm{Y}_{\mathrm{ij}}=$ the natural logarithm of the exposure concentration measured on the $j$-th day of the $i$-th worker

$\mu=$ the true underlying mean of log transformed exposure

$\beta_{1 . . \mathrm{k}}=$ fixed effects of $k$ exposure determinants (e.g. tasks, control measures)

$\chi_{\mathrm{i}}=$ random effect of the $i$-th worker

$\epsilon_{\mathrm{ij}}=$ random error (within-worker; day-to-day variation).

The model assumes that all $\chi_{\mathrm{i}}$, and $\epsilon_{\mathrm{ij}}$ are mutually independent and normally distributed with zero means and variances $\mathrm{Bw}_{\mathrm{y}}{ }^{2}$ and $\mathrm{ww}_{\mathrm{y}} \sigma_{\mathrm{y}}{ }^{2}$, representing between- and within-worker variability, respectively. These variances are estimated as between-worker $\left(\mathrm{BW}_{\mathrm{y}}{ }^{2}\right)$ and day-to-day $\left(w_{w} S_{y}{ }^{2}\right)$ covariance components by the restricted maximum likelihood (REML) algorithm.

To quantify the contribution of the fixed effects to the ${ }_{\mathrm{BW}} \mathrm{S}_{\mathrm{y}}{ }^{2}$ and ${ }_{\mathrm{ww}} \mathrm{S}_{\mathrm{y}}{ }^{2}$ covariance components, we compared the values of the covariance components obtained under model 1 with those from a "linear mixed-effects" model without the inclusion of fixed effects (in other words, one-way random effects model with only a random worker and day (error) component).

\section{Evaluation of grouping schemes}

Data from both surveys were used in the evaluation of the grouping schemes. Observations of workers with either a particulate or dermal exposure measurement missing for a given day were excluded, to enable direct comparisons between grouping schemes for these two different types of exposure. ${ }^{3}$ Due to the limited number of measurements in the department "laboratory", this department was excluded from these particular analyses. As a result the number of measurements reported in these analyses differ from the number of measurements reported in the exposure modelling analyses $(n=590$ versus 647 for dermal and 613 for inhalable dust, respectively). 
Table 2 Statistically significant exposure determinants $(p<0.10)$ of inhalable dust and dermal CSM exposure among workers in the rubber manufacturing industry

\begin{tabular}{|c|c|c|c|c|c|c|c|c|c|}
\hline \multicolumn{5}{|l|}{ Inhalable dust ( $n=613 ; k=226$ ) } & \multicolumn{4}{|l|}{ Dermal CSM ( $n=647 ; k=226)$} & \multirow[b]{2}{*}{$\mathbf{p}$} \\
\hline Determinant & $\mathbf{n}^{*}$ & $\beta \dagger$ & SE‡ & p§ & Determinant & $\mathbf{n}$ & $\boldsymbol{\beta}$ & SE & \\
\hline Intercept & & -0.51 & 0.05 & $<0.0001$ & Intercept & & 3.56 & 0.07 & $<0.0001$ \\
\hline \multicolumn{5}{|c|}{ Determinants related to relative higher exposure } & \multicolumn{5}{|c|}{ Determinants related to relative higher exposure } \\
\hline Weighing & 16 & 0.48 & 0.24 & 0.0490 & Weighing & 18 & 0.63 & 0.31 & 0.0396 \\
\hline Batch off & 12 & 1.16 & 0.43 & 0.0073 & Pouring chemicals & 12 & 0.96 & 0.36 & 0.0078 \\
\hline Extrusion of powdered rubber & 6 & 1.20 & 0.27 & $<0.0001$ & Cementing with brush & 18 & 0.84 & 0.31 & 0.0065 \\
\hline Jointing powdered products & 14 & 2.24 & 0.39 & $<0.0001$ & Jointing powered products & 14 & 0.83 & 0.37 & 0.0268 \\
\hline Autoclave powdered rubber & 6 & 1.06 & 0.40 & 0.0091 & Autoclave & 20 & 0.89 & 0.38 & 0.0205 \\
\hline Tube inspection & 6 & 1.42 & 0.44 & 0.0013 & UHF curing & 5 & 1.84 & 0.55 & 0.0008 \\
\hline Packing powdered products & 5 & 1.01 & 0.40 & 0.0124 & Tyre press & 26 & 1.95 & 0.51 & 0.0001 \\
\hline Packing & 36 & 0.39 & 0.17 & 0.0179 & Welding & 13 & 0.71 & 0.37 & 0.0561 \\
\hline Bench fitting & 20 & 1.33 & 0.26 & $<0.0001$ & Breakdown work & 38 & 0.81 & 0.24 & 0.0006 \\
\hline \multicolumn{5}{|c|}{ Determinants related to relative lower exposure } & \multicolumn{5}{|c|}{ Determinants related to relative lower exposure } \\
\hline Batch off with LEV & 7 & -0.85 & 0.51 & 0.0942 & Remixing & 12 & -0.68 & 0.38 & 0.0724 \\
\hline Calendering & 24 & -0.43 & 0.18 & 0.0181 & Manual assembling & 11 & -0.51 & 0.29 & 0.0733 \\
\hline Jointing & 36 & -0.41 & 0.24 & 0.0905 & Assembling machine & 23 & -0.87 & 0.37 & 0.0208 \\
\hline Laboratory & 9 & -0.93 & 0.41 & 0.0231 & Punching & 12 & -0.86 & 0.42 & 0.0390 \\
\hline \multirow{4}{*}{ Welding } & 13 & -1.09 & 0.32 & 0.0007 & Cement spraying with gloves & 18 & -0.90 & 0.39 & 0.0196 \\
\hline & & & & & Tyre press with gloves & 19 & -1.83 & 0.58 & 0.0017 \\
\hline & & & & & Autoclave with gloves & 11 & -0.89 & 0.50 & 0.0759 \\
\hline & & & & & Inspection & 47 & -0.42 & 0.20 & 0.0409 \\
\hline Supervision & 62 & -0.38 & 0.13 & 0.0030 & Supervision & 64 & -0.39 & 0.17 & 0.0204 \\
\hline Variance & & Total $^{\star *}$ & Modeltt & $\% \ddagger \ddagger$ & Variance & & Total & Model & $\%$ \\
\hline$b_{w} S_{y}{ }^{2} \S \S$ & & 0.60 & 0.38 & 37 & $b_{w} S_{y}^{2}$ & & 0.64 & 0.38 & 41 \\
\hline$w_{w} S_{y}^{2} q$ & & 0.38 & 0.34 & 11 & $w w S_{y}^{2}$ & & 0.85 & 0.85 & 0 \\
\hline \multicolumn{10}{|c|}{$\begin{array}{l}\text { *Number of times determinant was present. } \\
\text { †Regression coefficient estimate. } \\
\text { †Standard error of estimate. } \\
\S p \text { value. } \\
\text {-Compared to the true underlying mean exposure (intercept). } \\
\text { **Total variance from model with only random effects. } \\
\dagger † V a r i a n c e \text { from model with fixed and random effects. } \\
\text { ††\% of variance explained by fixed effects. } \\
\S \S \text { Estimated variance of the distribution of logarithmic means } \\
\text { - Estimated variance of the distribution of logarithmic means }\end{array}$} \\
\hline
\end{tabular}

Four different grouping schemes were created that were based on: (1) plant ( $\mathrm{g}=9$ ); (2) type of plant ( $\mathrm{g}=3$; rubber tyre and belt production, retreading, and general rubber goods); (3) department ( $\mathrm{g}=7$; compounding, pre-treating, moulding, curing, finishing, shipping, engineering service); and (4) a determinant based exposure grouping ( $\mathrm{g}=3$; low, medium, high exposure). In addition, combinations of these groupings were explored as well: (5) plant/department $(\mathrm{g}=56)$; (6) plant/ determinant based ( $\mathrm{g}=25$ or 26 , for inhalable dust and dermal exposure, respectively); (7) department/determinant based $(\mathrm{g}=2 \mathrm{l}$ or 20 , for inhalable dust and dermal exposure, respectively). The determinant based exposure groups (low, medium, and high) were based on the identified exposure determinants within the 1997 survey using the procedure described by Kromhout and Heederik. ${ }^{3}$ If a subject performed a task on the measured shift that was related to relative higher exposures, he or she was assigned to the high exposure group for that day. If a subject did not perform tasks related to higher exposures but performed a task related to relative lower exposure levels, he or she was assigned to the low exposure category for that day. All others who performed a task related to neither higher or lower exposures were assigned to the medium exposure group for that day. None of the study subjects performed tasks within a day that were related to both relative higher and lower exposures. Furthermore, most subjects did not change exposure categorisation between measurement days. Subjects that changed exposure categories between measurement days ( $16 \%$ for inhalable dust and 18\% for dermal exposure) were assigned to their definitive low, medium, or high exposure group category based on the predominant daily exposure group assignment for that particular subject.

The within- and between-worker components of exposure variance were estimated using multiple linear mixed models. In these models, grouping variables were treated as fixed effects and worker identity was introduced as a random effect. ${ }^{13}$ Note, these models are essentially the same as equation 1 except for the definition of the fixed effect (exposure determinants versus exposure grouping). Between-worker variance component was used to estimate the range within which $95 \%$ of the individual mean exposure fall $\left({ }_{b w} R_{0.95}=\exp ^{(3.92 b w S y)}\right)$. Correspondingly, the withinworker variance component was used to estimate the range within which $95 \%$ of the day-to-day estimates of exposure for an individual fall $\left({ }_{w w} R_{0.95}=\exp ^{(3.92 \text { wwSy })}\right) \cdot{ }^{14}$

Average expected contrast in exposure estimates $(\epsilon)$ was estimated for each grouping as a ratio of the between-group variance estimate and the sum of the between and withingroup variance estimates: $\epsilon={ }_{\mathrm{bg}} \mathrm{S}_{\mathrm{Y}}{ }^{2} /\left(\mathrm{bg}_{\mathrm{y}} \mathrm{S}^{2}+{ }_{\mathrm{wg}} \mathrm{S}_{\mathrm{y}}{ }^{2}\right)$. The between-group variance was estimated as the difference between ${ }_{\mathrm{bw}} \mathrm{S}_{\mathrm{y}}{ }^{2}$ for the model with random effect worker only and ${ }_{b w} S_{y}{ }^{2}$ for the model with both the fixed grouping and random worker effects. The sum of the between- and withingroup variances for a model with a grouping variable (fixed effect) was estimated as ${ }_{b w} \mathrm{~S}_{\mathrm{y}}{ }^{2}$ for the model with random worker effect only. This is only a correct approximation if the grouping variable has no impact on ${ }_{\mathrm{ww}} \mathrm{S}_{\mathrm{Y}}{ }^{2} \cdot{ }^{13}$ This assumption was met in our data, as workers did not change grouping status (by design) between their respective repeat measurements. 
Least square mean estimates of inhalable dust and dermal CSM for each exposure group in the explored grouping schemes were calculated based on both the 1997 and 1988 exposure data using the described mixed models. Subsequently, the $1997\left(\mathrm{Y}_{\mathrm{gi}, 97}\right)$ and $1988\left(\mathrm{Y}_{\mathrm{gi}, 88}\right)$ group estimates were compared. Bias was defined as the mean difference between the least square mean estimates in logspace between the individual group estimates $\left(\mathrm{Y}_{\mathrm{gi}, 88}-\mathrm{Y}_{\mathrm{gi}, 97}\right)$ and precision as the standard deviation of the difference in these individual group estimates. The exponent of the calculated bias $\left(\exp \left(\mathrm{Y}_{\mathrm{gi}, 88}-\mathrm{Y}_{\mathrm{gi}, 97}\right)\right)$ provides the ratio between the 1988 and 1997 exposure estimates on the original scale. Correlations between the 1988 and 1997 least square mean group estimates by grouping scheme were calculated using Spearman correlation statistics. The latter analyses were limited to the seven plants that were represented in both surveys.

All statistical analyses were performed with SAS 8.2 (SAS Institute), utilising proc GLM, proc MIXED, or proc CORR.

\section{RESULTS}

\section{Identification of exposure determinants}

In 1997, the 8-hour TWA geometric mean inhalable dust concentration varied from 0.4 to $0.9 \mathrm{mg} / \mathrm{m}^{3}$ and from 0.2 to $1.0 \mathrm{mg} / \mathrm{m}^{3}$ when analysed by plant and department, respectively (table 1). Inhalable dust exposure per type of industry was reasonably comparable across the different classifications (range $0.6-0.8 \mathrm{mg} / \mathrm{m}^{3}$ ). The linear mixed model revealed several exposure determinants that were related to relative higher $(n=9)$ and lower $(n=6)$ inhalable dust concentrations (table 2). Higher dust exposures were mostly related to the handling of powdered rubber parts and weighing of chemicals. Identified exposure determinants explained $37 \%$ and $11 \%$ of the between- and within-worker variance, respectively.

The geometric mean dermal CSM concentrations varied from 26 to $50 \mu \mathrm{g} / \mathrm{cm}^{2}$ and from 26 to $68 \mu \mathrm{g} / \mathrm{cm}^{2}$ when analysed by plant and department, respectively (table 1). In total nine exposure determinants were associated with higher dermal exposure levels and nine exposure determinants were associated with lower dermal exposure levels. Higher dermal exposure levels were predominantly related to curing and maintenance work. Identified exposure determinants explained $41 \%$ of the between-worker variance, but did not explain day-to-day variance.

\section{Evaluation of grouping schemes}

Seven different grouping schemes commonly used in epidemiological studies in the rubber manufacturing industry were evaluated (table 3). The model with only the worker's identity as random effect revealed that across the industry the between-worker variance was larger than the day-to-day variance for inhalable dust exposure. For dermal exposure the opposite was found with a substantial day-to-day variability in exposure levels as indicated by the large $R_{0.95}$ fold range of the within person exposure distribution $\left({ }_{w w} R_{0.95}=35.6\right)$.

For inhalable dust exposure, it is clear from the data presented in table 3 that grouping based solely on observational factors (for example, plant, type of plant, and departments) resulted in poor contrast (0.00-0.05). Combining plant and departments increased the contrast to 0.16. Contrast for the exposure groupings based on the identified determinants performed better, but resulted still in only a moderate contrast $(\epsilon=0.28)$. Combination of this classification with plant or department resulted in a clear increase in contrast with the combination of plant and exposure determinants as the most favourable grouping $(\epsilon=0.49)$.

The contrast of the different grouping schemes for dermal exposure was comparable to those for inhalable exposure with plant and departments resulting in low contrast (range $0.00-0.25)$ and determinant based groupings resulting in a higher contrast $(\epsilon=0.34)$. However, combination of the determinant based grouping with plant and departments did not result in an increase in contrast. All grouping schemes still revealed a considerable difference in average dermal exposure levels between workers within groups $\left(_{b w} \mathrm{R}_{0.95}{ }_{13-}\right.$ 24).

The same groupings were applied to the exposure data collected during the 1988 survey. For inhalable dust the performance of the commonly used plant and department groupings are more or less comparable to the results based on the 1997 survey (table 4). However, the determinant based classification derived from the 1997 exposure survey performed less well in the 1988 survey as for the 1997 data itself $\left(\epsilon_{88}=0.11\right.$ versus $\left.\epsilon_{97}=0.28\right)$. The same was observed for dermal CSM exposure where the contrast of the determinant based grouping was also reduced in the 1988 survey compared to the 1997 survey $\left(\epsilon_{88}=0.14\right.$ versus $\left.\epsilon_{97}=0.34\right)$. The combination of plant and department showed the best contrast in the 1988 exposure data for both inhalable dust and dermal CSM levels and in both cases actually increased when compared to the 1997 survey.

Bias in group estimates was considerable with estimates of average exposure based on 1988 data about one-and-a-half to two-fold higher than those extrapolated from the 1997 data. Overall precision was worse for grouping schemes with more individual groups (for example, plant and department). Spearman correlation between the estimates for the different

\begin{tabular}{|c|c|c|c|c|c|c|c|c|c|}
\hline \multirow[b]{2}{*}{ Grouping variable } & \multirow[b]{2}{*}{ No. of groups } & \multicolumn{4}{|c|}{ Inhalable dust } & \multicolumn{4}{|c|}{ Dermal CSM } \\
\hline & & $\mathrm{bg}^{2{ }^{2 *}}$ & ${ }_{b w} S_{y}{ }^{2} \dagger$ & $w w S_{y}{ }^{2} \ddagger$ & $\epsilon \S$ & $\mathrm{bg} \mathrm{S}_{y}{ }^{2}$ & $b_{w} S_{y}{ }^{2}$ & $w w S_{y}{ }^{2}$ & $\epsilon$ \\
\hline Worker only & & NA & 0.59 & 0.38 & & NA & 0.66 & 0.83 & \\
\hline Department & 7 & 0.01 & 0.57 & 0.38 & 0.02 & 0.04 & 0.62 & 0.83 & 0.06 \\
\hline Plant & 9 & 0.03 & 0.56 & 0.38 & 0.05 & 0.02 & 0.65 & 0.84 & 0.03 \\
\hline Type & 3 & 0.00 & 0.58 & 0.38 & 0.00 & 0.00 & 0.66 & 0.83 & 0.00 \\
\hline Determinant based & 3 & 0.16 & 0.42 & 0.38 & 0.28 & 0.23 & 0.44 & 0.84 & 0.34 \\
\hline Plant/department & 56 & 0.10 & 0.49 & 0.38 & 0.16 & 0.16 & 0.50 & 0.84 & 0.25 \\
\hline Plant/determinant based & $25 / 26$ & 0.29 & 0.30 & 0.38 & 0.49 & 0.21 & 0.45 & 0.84 & 0.32 \\
\hline Department/determinant based & $21 / 20$ & 0.24 & 0.35 & 0.38 & 0.40 & 0.19 & 0.48 & 0.84 & 0.28 \\
\hline $\begin{array}{l}\text { *Between-group variance. } \\
\text { †Between-worker variance or wi } \\
\text { †Within-worker variance. } \\
\text { \$Contrast between groups: estim } \\
\text { - Number of groups for inhalable }\end{array}$ & $\begin{array}{l}\text { up variance. } \\
\text { etween-group } \\
\text { ermal exposur }\end{array}$ & $\begin{array}{l}\text { nce/s } \\
\text { pecti }\end{array}$ & setwee & within & p vc & & & & \\
\hline
\end{tabular}


Table 4 Contrast, bias, precision, and Spearman correlation between the least square mean estimates for the different groups based on the 1988 and 1997 exposure survey

\begin{tabular}{|c|c|c|c|c|c|c|}
\hline \multirow[b]{2}{*}{ Grouping variable } & \multicolumn{2}{|c|}{ Contrast } & \multicolumn{4}{|c|}{ Comparison of groups* } \\
\hline & $\epsilon 97 \dagger$ & $\epsilon \mathbf{8 8} \ddagger$ & $\begin{array}{l}\text { No. of } \\
\text { groups }\end{array}$ & Bias§ & Precision ฯ & $\begin{array}{l}\text { Correlation } \\
\text { Spearman }\end{array}$ \\
\hline \multicolumn{7}{|l|}{ Inhalable dust } \\
\hline Department & 0.02 & 0.06 & 7 & 0.47 & 0.22 & 0.29 \\
\hline Plant & 0.05 & 0.00 & 7 & 0.44 & 0.26 & 0.39 \\
\hline Type & 0.00 & 0.00 & 3 & 0.49 & 0.18 & 0.50 \\
\hline Determinant based** & 0.28 & 0.11 & 3 & 0.53 & 0.26 & 0.50 \\
\hline Plant/department & 0.16 & 0.27 & 38 & 0.53 & 0.97 & 0.53 \\
\hline Plant/determinant based & 0.49 & 0.19 & 20 & 0.50 & 0.61 & 0.47 \\
\hline Department/determinant based & 0.40 & 0.18 & 19 & 0.48 & 0.42 & 0.62 \\
\hline \multicolumn{7}{|l|}{ Dermal CSM } \\
\hline Department & 0.06 & 0.18 & 7 & 0.67 & 0.32 & 0.71 \\
\hline Plant & 0.03 & 0.01 & 7 & 0.61 & 0.33 & -0.29 \\
\hline Type & 0.00 & 0.00 & 3 & 0.76 & 0.12 & 0.50 \\
\hline Determinant based** & 0.34 & 0.14 & 3 & 0.74 & 0.26 & 1.00 \\
\hline Plant/department & 0.25 & 0.32 & 38 & 0.64 & 0.93 & 0.55 \\
\hline Plant/determinant based & 0.32 & 0.20 & 20 & 0.67 & 0.76 & 0.45 \\
\hline Department/determinant based & 0.28 & 0.20 & 19 & 0.67 & 0.47 & 0.64 \\
\hline \multicolumn{7}{|c|}{$\begin{array}{l}\text { *Comparison of groups was limited to data from the seven plants that were represented in both surveys. } \\
\text { †Contrast between groups based on exposure data from the } 1997 \text { exposure survey }(n=590 ; k=220) \text {. } \\
\text { †Contrast between groups based on exposure data from the } 1988 \text { exposure survey }(n=591 ; k=244) \text {. } \\
\text { \$Mean difference between the } 1988 \text { and } 1997 \text { estimates in log-space of the individual group estimates; the } \\
\text { exponent of the calculated bias (exp }\left(Y_{\text {gi, } 88}-Y_{\text {gi,97 }}\right) \text { provides the ratio between the } 1997 \text { and } 1988 \text { estimates on } \\
\text { the original scale. } \\
\text { TStandard deviation in the difference in individual group estimates based on the } 1988 \text { and } 1997 \text { survey. } \\
\text { **Determinant based grouping is based on exposure determinants identified in the } 1997 \text { survey. }\end{array}$} \\
\hline
\end{tabular}

groups based on the two surveys varied considerably with a relative high correlation for the determinant based grouping and the grouping based on the combination of plant and department.

\section{DISCUSSION}

Grouping workers into exposure categories is a common necessity in many occupational epidemiological studies. Such groupings can be based on commonly used observational characteristics like plant, department, etc. It has been hypothesised that by constructing exposure groups based on identified exposure determinants, more optimal groupings can be derived. ${ }^{311}$ We used data from two cross-sectional industry wide exposure surveys conducted in 1997 and 1988 in the rubber manufacturing industry to explore the validity of using groupings based on identified determinants. In essence these analyses can be seen as exposure assessment validation by using an independent (at least in time) dataset. As such these analyses go beyond internal validation by bootstrapping or by splitting the dataset into a model development and model validation set.

Several determinants were identified that were related to higher dust exposure levels. Most of these determinants were related to the handling of powdered rubber parts and weighing of chemicals. The majority of these determinants were also identified in the 1988 survey with only "batch off" uniquely identified in the1997 survey and "repair buffing" in the 1988 data. ${ }^{10}$ The overlap in determinants related to lower inhalable dust exposures was much less between the two surveys, with only "laboratory work" being identified in both surveys. These results did not change notably when only data from the seven overlapping companies were used to identify possible exposure determinants in the 1997 dataset (results not shown). Higher dermal exposure levels were predominantly related to curing and maintenance work. Of the nine determinants related to higher dermal exposure levels, six (weighing, autoclave, tyre press, pouring chemicals, welding, and breakdown work) were also identified in the 1988 survey. ${ }^{10}$ Again determinants related to lower exposures were found to be much less consistent between the two surveys, with only supervision being identified in both surveys.

The identified determinants were subsequently used to construct determinant based exposure groupings based on the 1997 survey as described previously by Kromhout and Heederik. ${ }^{3}$ Alternatively, other exposure groupings were used based on plant and department. For both inhalable dust exposure and dermal CSM exposure, the highest contrast was observed for the grouping based on identified exposure determinants, which is consistent with the results reported by Kromhout and Heederik. ${ }^{3}$ Nevertheless, the contrast that was achieved by this grouping scheme was only moderate $(\epsilon<0.50)$, and considerable differences up to a factor of 10$15\left({ }_{b w} R_{0.95}\right)$ in average exposures between subjects within groups remained. Based on these results a classification scheme based on plant and identified determinants would overall be preferable. It has to be noted however that this decision is based entirely on the quantitative aspects of the exposures. However, we have previously shown, based on mutagenicity tests of both air and surface samples, that the qualitative aspects of these exposures are highly variable, because the chemicals used and intermediates produced during the processes are multitudinous. ${ }^{15}{ }^{16}$ Moreover, it is clear from our analyses that the difference between plants could not be fully explained by differences in production processes and/or tasks performed. If the plant effect cannot be explained by observable factors, then such a classification has no value outside the studied plants.

The contrast of the different observational grouping schemes based on plant and department within the 1997 exposure survey was reasonably similar to those of the 1988 survey. However, when the classification based on the identified determinants in the 1997 survey was used in the 1988 survey, a considerable decrease in contrast was observed for both inhalable and dermal CSM exposure. This was also observed in the opposite direction, meaning that if the determinant based grouping of 1988 as described by Kromhout and Heederik ${ }^{3}$ was superimposed on the 1997 data, a similar decrease in contrast was observed for both 


\section{Main messages}

- Grouping subjects by departments results in poor contrast for both inhalable dust and dermal CSM exposure.

- Determined based exposure groupings lead indeed to more efficient groupings within a cross-sectional survey itself, but such groupings might have significantly less contrast if applied retrospectively.

inhalable dust $\left(\epsilon_{88}=0.21 ; \epsilon_{97}=0.12\right)$ and dermal CSM exposure $\left(\epsilon_{88}=0.20 ; \epsilon_{97}=0.12\right)$. As we observed that the determinants associated with relative higher dust and dermal exposures were more consistent over time than the exposure determinants related to relative lower exposures, we explored an alternative determinant based grouping by classifying subjects into a high and a low (previous medium and low) exposure group. Contrast of this grouping was essentially similar to the original three group classification and again showed a similar drop in contrast when applied to the other dataset (results not shown). It is clear from these results that determinant based groupings lead to more efficient groupings within a cross-sectional survey itself, but one should be careful to use these groupings outside the studied population and time period.

The predicted exposures for 1988 based on the 1997 data were lower than estimates derived from the 1988 data itself. This is not surprising as we previously found that exposure levels within the rubber industry in the Netherlands have decreased on average with $5.7 \%$ and $6.7 \%$ per year for inhalable dust and dermal exposure, respectively. ${ }^{8}$ Bias was overall similar between the different groupings; however, precision differed largely with not surprisingly the worst precision for grouping schemes with the largest number of groups. Relative ranking between the groupings based on the 1997 and 1988 surveys were most consistent for the classifications based on the combination of plant and department and for the classification based on the combination of department and determinants. From these results it is not instantly clear what the best grouping scheme would be for retrospective epidemiological research in the rubber industry. If exposure information is available on all plants and departments in the epidemiological study, a classification based on plant and department or plant and determinants seems to be the most appropriate as it results in the best overall contrast and the classification seems to preserve its relative ranking over time. However, if exposure information is not available for each plant in the epidemiological study and therefore "plant" cannot be used as a grouping variable, the exposure grouping has to rely on a combination of department and exposure determinants. It is evident that relying solely on departments in epidemiological research in the rubber industry results in poor contrast for both inhalable dust and dermal CSM exposure. Although determinant based groupings seem to improve contrast, they have to be used with caution as they might have significantly less contrast beyond the studied population or time period. This is especially true when considering that seven factories participated in both surveys and that production and layout of the factories were largely identical within both surveys. ${ }^{8}$

The results of this study of course pertain to the situation that a current or single survey is used to identify determinants, which are then used in a broader setting than they were originally derived from. Collecting historical data covering the whole time period and identifying exposure affecting determinants within the whole dataset might have

\section{Policy implications}

- A classification based on plant and department might be more desirable for retrospective studies in the rubber manufacturing industry, as they seem to have more historical relevance and are most likely more accurately recorded historically than information on particular exposure determinants in a particular industry. Exposure determinants might not have relevance beyond the period where they are based.

- Longitudinal collected measurement data is needed in order to construct exposure groups that potentially have better and more consistent contrast over time.

revealed determinants that could have led to a grouping which showed a better and consistent contrast over time. ${ }^{17}{ }^{18}$ It is however clear that given the large bias, historical exposure trends have to be incorporated in retrospective exposure estimates, which can only be achieved by collecting longitudinal exposure data.

It can of course be debated whether these results can be generalised to other occupational settings. Clearly, the exposure range in inhalable dust and dermal CSM within the rubber industry is relatively small compared to (other) exposures in other industries, which would make any exposure grouping difficult and more likely to be unstable. Furthermore, we know that exposures in the rubber industry have changed considerably in recent decades ${ }^{8}$ and as such it could have been expected that using a contemporary survey to group workers might not hold up over time. However, decreasing occupational exposures over time seem to be more the norm than an exception, ${ }^{19}$ and as such the observed situation here might not be different than for many other occupational settings. Thus, our results give some pause to the use of cross-sectional surveys to optimise exposure groupings and for the use of detailed information on exposure determinants as they might not hold up over time. Similar analyses in other occupational settings should be performed to determine the generalisability of this observation. This exercise however emphasises the need to characterise exposure variability when constructing exposure groups, to quantify their efficiency, and stresses the need for longitudinal collected measurement data.

\section{Authors' affiliations}

R Vermeulen, Occupational and Environmental Epidemiology Branch, Division of Cancer Epidemiology and Genetics, National Cancer Institute, Rockville, MD, USA

H Kromhout, Environmental and Occupational Health Group, Institute of Risk Assessment Sciences, Utrecht University, Utrecht, Netherlands

Competing interests: none declared

\section{REFERENCES}

1 Ahrens W, Stewart T. Retrospective exposure assessment. In: Nieuwenhuijsen $M$, eds. Exposure assessment in occupational and environmental epidemiology. New York: Oxford University Press, 2003:103-18.

2 Prais SJ, Aitchinson J. The grouping of observations in regression analysis. Rev Int Stat Inst 1954; 1(3): 1-22.

3 Kromhout H, Heederik D. Occupational epidemiology in the rubber industry: implications of exposure variability. Am J Ind Med 1995;27:171-85.

4 Seixas NS, Sheppard L. Maximizing accuracy and precision using individual and grouped exposure assessments. Scand J Work Environ Health 1996;22:94-101.

5 Kromhout $\mathbf{H}$, Loomis DP. The need for exposure grouping strategies in studies of magnetic fields and childhood leukemia. Epidemiology 1997;8:218-19.

6 Houba R, Heederik D, Kromhout H. Grouping strategies for exposure to inhalable dust, wheat allergens and alpha-amylase allergens in bakeries. Ann Occup Hyg 1997;41:287-96. 
7 van Tongeren M, Gardiner K, Calvert I, et al. Efficiency of different grouping schemes for dust exposure in the European carbon black respiratory morbidity study. Occup Environ Med 1997;54:714-19.

8 Vermeulen R, de Hartog J, Swuste P, et al. Trends in exposure to inhalable particulate and dermal contamination in the rubber manufacturing industry: effectiveness of control measures implemented over a nine-year period. Ann Occup Hyg 2000;44 343-54.

9 Swuste P, Kromhout H, Drown D. Prevention and control of chemical exposures in the rubber manufacturing industry in the Netherlands. Ann Occup Hyg 1993;37:1 17-34.

10 Kromhout $H$, Swuste P, Boleij JS. Empirical modelling of chemical exposure in the rubber-manufacturing industry. Ann Occup Hyg 1994:38:3-22

11 Peretz C, Goren A, Smid T, et al. Application of mixed-effects models for exposure assessment. Ann Occup Hyg 2002;46:69-77.

12 Kenny LC, Aitken R, Chalmers C, et al. A collaborative European study of personal inhalable aerosol sampler performance. Ann Occup Hyg 1997;41:135-53.
13 Burstyn I, Kromhout $\mathrm{H}$. Are the members of a paving crew uniformly exposed to bitumen fume, organic vapor, and benzo(a)pyrene? Risk Anal 2000;20:653-63.

14 Rappaport SM, Kromhout H, Symanski E. Variation of exposure between workers in homogeneous exposure groups. Am Ind Hyg Assoc J 1993;54:654-62

15 Vermeulen R, Bos RP, de Hartog J, et al. Mutagenic profile of rubber dust and fume exposure in two rubber tire companies. Mutat Res 2000:468:165-71.

16 Vermeulen R, Bos RP, Kromhout H. Mutagenic exposure in the rubber manufacturing industry: an industry wide survey. Mutat Res 2001;490:27-34

17 Burstyn I, Kromhout H, Cruise PJ, et al. Designing an international industrial hygiene database of exposures among workers in the asphalt industry. Ann Occup Hyg 2000;44:57-66.

18 Burstyn I, Kromhout H, Kauppinen T, et al. Statistical modelling of the determinants of historical exposure to bitumen and polycyclic aromatic hydrocarbons among paving workers. Ann Occup Hyg 2000;44:43-56.

19 Kromhout H, Vermeulen R. Long-term trends in occupational exposure: are they real? What causes them? What shall we do with them? Ann Occup Hyg 2000;44:325-7.

\section{$\mathrm{ECHO}$}

\section{Genes for antioxidant enzymes and PMF are not linked}

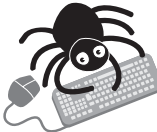

Please visit the Occupational and

Environmental Medicine website [www. occenvmed. com] for a link to the full text of this article.
- usceptibility to progressive massive fibrosis (PMF) and polymorphisms in genes for antioxidant enzymes are not associated, according to the first case-control study to test $\checkmark$ this out. However, polymorphisms combined with environmental factors might still affect severity of the disease-a severe form of coal workers' pneumoconiosis.

Common single polymorphic variants of glutathione S-transferase (GST) and manganese superoxide dismutase (MnSOD)_GSTP1, GSTT1, and MnSOD_were not statistically associated with PMF in 350 ex-underground coal workers compared with control miners matched for age, years of mining exposure, and smoking but with no evidence of lung fibrosis or inflammation. Nor were there any significant gene-gene interactions, which would be expected for a multifactorial disease like PMF. Frequencies of the polymorphisms conformed to the Hardy-Weinberg equilibrium in both groups. The study was powerful enough to detect a relation between susceptibility and polymorphic genes at an odds ratio of 1.8 .

Histologically confirmed cases of PMF were identified from a well defined group of miners taking part in the national coal workers autopsy study in the United States during 1972-96, from whom necroscopic lung samples had been collected. Genetic analysis relied on DNA extracted and amplified from these samples.

Genes for antioxidant GSTs and superoxide dismutases MnSOD, which combat the harmful effects of reactive oxygen species in the lungs and help to protect against interstitial lung disease, are very polymorphic. Several common variants of GSTs have been associated with cancers of various organs, including lungs, and chronic obstructive pulmonary disease.

$\Delta$ Yucesoy B, et al. Thorax 2005;60:492-495. 